\section{Origins of senescence}

The Genetics of Aging. Edited by E. L. Schneider. Pp.424. (Plenum: New York and London, 1978.) \$39.

Despite a considerable amount of research in recent years, as one of the contributors to this volume states, “. . . a universally acceptable definition of normal biological aging is still not available. The origins of senescence remain enigmatic, and little agreement exists regarding its true nature". Having read this well referenced up-to-date review of the subject one cannot help but agree with this sentiment, but as the nature and cause of aging raise basic fundamental questions in biology perhaps this is not too surprising.

The book is divided into four parts. The first is concerned with the possible molecular basis of aging which resolves essentially into error and programmed theories. The former assumes that aging results from the accumulation of errors in DNA replication or deficiencies in the repair of such errors. The latter assumes that aging results from genetically programmed senescence. Evidence is presented both for and against these two possibilities. The observation in man of increasing hypodiploidy in cultured lymphocytes with age, most frequently affecting the $X$ chromosome in females and the $Y$ chromosome in males, is intriguing, but whether this is a cause or effect of aging remains unresolved.

The second part of the book is concerned with aging at the level of the organism itself from protozoa and fungi to vertebrates. Whether certain findings in lower organisms are of general relevance is a mute point. For example, in Podospora anserina (a filamentous fungus) there is clear evidence that longevity is at least partly controlled by cytoplasmic factors. In higher forms life span and survivorship can be expressed in terms of allometric and Gompertz equations for which Sacher presents convincing explanations. It would seem reasonable, however, that aging in man is at least partly determined by genetic factors and the third and largest part of the book is devoted to this subject. In 1961 Hayflick first demonstrated that human diploid somatic cells have a limited replicative life span in tissue culture which is inversely related to the age of the donor. Since then, this has become an exciting and popular experimental tool for studying the phenommenon of aging at least as this is expressed in vitro. Detailed consideration is given to this phenomenon in the case of those genetic syndromes known to be associated with premature aging such as progeria in which fibroblasts in culture show reduced survival, defective HLA expression, increased heat lability of cytoplasmic enzymes, changes in insulin receptors and tissue factors promoting clotting. These observations are appealing; but are such disorders a legitimate paradigm of normal aging? The possible role of genetic factors in aging is more directly approached by family and twin studies. The findings in the now famous Pearl (Baltimore) study of longevity among relatives of nonagenarians are discussed in detail and indicate that there is a definite familial component in aging. However, this is small and Murphy is careful to avoid being dogmatic as to its nature. It could well be largely cultural or environmental. On the other hand, the discussion of

\section{Nuclear transplantation experiments in Amphibia}

Cloning: Nuclear Transplantation in Amphibia. By R. G. McKinnell. (University of Minnesota Press: Minneapolis, 1978.) $\$ 22.50$.

IN spite of its principal title, this book is not concerned with most aspects of cloning. Thus, it does not deal with cloning in plants, with the cloning of DNA or genes in bacteria by plasmids and phages, nor with the cloning of animal cells in culture. In fact the book consists entirely of an account of nuclear transplantation experiments in Amphibia, primarily those with Rana pipiens. This has its use, and a chapter on the transplantation of nuclei from tumour cells is clearly presented and well balanced.

Unfortunately the same cannot be said for much of the rest of the book. In general, work with species other than $R$. pipiens is treated as trivial or at best confirmatory. Often it is hard to discern the direction of an argument. Thus, the book does not make it clear that many amphibian nuclear transfer experiments have been addressed to the specific question of whether the nucleus of one kind of specialised cell contains the genetic information necessary to promote the formation of other quite unrelated cell types. Though generally considered important, this question is totally obscured in this book by the author's decision to emphasise the age rather than differentiated state of donor cells.

From the student's point of view, a twin studies gives more emphasis to a genetic component in aging.

The fourth and final part of the book is concerned with certain experimental approaches to aging, including somatic cell genetics, immunogenetics and behavioural genetics.

Inevitably in a work of this nature there is some repetition but this is less than in most large multi-authored books. In any event it is often interesting to compare the views of different contributors on the same subject; though the index itself is often unhelpful in this regard. This is a highly readable and well referenced review of a fascinating subject and $I$ would recommend it without reservation.

Alan E. H. Emery

Alan E. H. Emery is Professor of Human Genetics at the University of Edinburgh, $U K$.

serious deficiency is that alternative but not exclusive interpretations are often omitted. For example, most of the first half of the book is concerned with abnormal embryos resulting from nuclear transfers in $R$. pipiens. It is therefore disappointing to find that one of the principal ideas regarding the significance of these abnormal embryos is entirely overlooked. The author presses the view that the abnormalities of nuclear transplant embryos may give specific information about the specialised state of gene control in donor cells. An alternative interpretation (favoured by several in this field) is that abnormal embryos result from random chromosomal losses due to the low replication rate of specialised cells and the very high rate at which eggs always divide. My criticism is not that the author subscribes to the former point of view, but to disguise or ignore the second interpretation seems to me to significantly misrepresent the literature.

The last half of the book contains useful technical information in the form of six appendices. These deal with the biology and maintenance of $R$. pipiens, the instruments, solutions and techniques used to transplant single nuclei to eggs (primarily $R$. pipiens), as well as with sources of various anuran species.

The unbalanced presentation and uncritical interpretation of nuclear transplantation experiments seriously limit the general usefulness of the first part of this book. However, the technical information in the appendices should prove valuable for those wishing to transplant nuclei in $R$. pipiens.

\section{J. B. Gurdon} of Molecular Biology in Cambridge, UK. 